\title{
Breaking Bad News: Patient Preferences and the Role of Family Members when Delivering a Cancer Diagnosis
}

\author{
Abha Rao ${ }^{1}$, Bhuvana Sunil ${ }^{1}$, Maria Ekstrand ${ }^{3,1}$, Elsa Heylen $^{3}$, Girish Raju $^{2}$, Arun Shet $^{1,2 *}$
}

\begin{abstract}
Background: Western physicians tend to favour complete disclosure of a cancer diagnosis to the patient, while non-Western physicians tend to limit disclosure and include families in the process; the latter approach is prevalent in clinical oncology practice in India. Few studies, however, have examined patient preferences with respect to disclosure or the role of family members in the process. Materials and Methods: Structured interviews were conducted with patients $(\mathrm{N}=127)$ in the medical oncology clinic of a tertiary referral hospital in Bangalore, India. Results: Patients ranged in age from 18-88 $(\mathrm{M=52})$ and were mostly male $(59 \%)$. Most patients $(\mathbf{7 2 \%})$ wanted disclosure of the diagnosis cancer, a preference significantly associated with higher education and English proficiency. A majority wanted their families to be involved in the process. Patients who had wanted and not wanted disclosure differed with respect to their preferences regarding the particulars of disclosure (timing, approach, individuals involved, role of family members). Almost all patients wanted more information concerning their condition, about immediate medical issues such as treatments or side effects, rather than long-term or non-medical issues. Conclusions: While most cancer patients wanted disclosure of their disease, a smaller group wished that their cancer diagnosis had not been disclosed to them. Regardless of this difference in desire for disclosure, both groups sought similar specific information regarding their cancer and largely favoured involvement of close family in decision making. Additional studies evaluating the influence of factors such as disease stage or family relationships could help guide physicians when breaking bad news.
\end{abstract}

Keywords: Cancer disclosure - patient preferences - role of families - India

Asian Pac J Cancer Prev, 17 (4), 1779-1784

\section{Introduction}

Disclosure in the context of oncology care refers to the act of divulging information to a patient (or his or her family members) about a diagnosis of cancer, the prospect of recovery, or any other consequences resulting from the usual course of cancer or its treatment. Research suggests that it is important to disclose diagnostic and prognostic information about cancer, since in the absence of effective communication patients tend to over-estimate their chances of survival, leading to poor decision-making regarding therapeutic interventions and care (Russell and Ward, 2011). On the other hand, the documented benefits of disclosure are considerable: patients and family members are emotionally and practically better prepared, trust and better communication between patient and doctor are improved, and patients are involved in treatment decisions and experience lower levels of anxiety and depression, symptom control and satisfaction with care (Lee and Wu, 2002; Russell and Ward, 2011).

Historically, the medical profession was paternalistic with regard to disclosure: the doctor generally made the decision, on behalf of the patient, about whether to reveal a diagnosis, and if so, how much to reveal about it. The doctor's decision was typically driven by the need to balance beneficence and veracity (Mitchell, 1998; Kazdaglis et al., 2010). Over time, evolution in legal conceptions of patient rights and informed consent (Searight and Gafford, 2005), or advances in cancer treatment, which require the patient's knowledge and cooperation (Kazdaglis et al., 2010), have resulted in changing practices, particularly in the west. Patient rights are less salient in most non-Western countries, perhaps due to the unequal power dynamic between doctors and patients, the enormous time and resource constraints on doctors (Ghooi and Deshpande, 2012), or cultural limitations with respect to understanding what patient rights mean (Masaki et al., 2014). Doctors may also be poorly trained or inadequately prepared for the disclosure of a cancer diagnosis to the patient (Grassi et al., 2000; Kumar et al., 2009).

Disclosure can be guided by cultural norms: some 
cultures view discussing the cancer patient's condition directly with the patient as inappropriate and even cruel (the 'community-oriented' east), while in other cultures, individual autonomy is valued over all else (the 'individualist' west) (Mitchell, 1998; Chaturvedi et al., 2009). A number of literature reviews reveal further nuances in the way doctors (Grassi et al., 2000; Kumar et al., 2009), patients (Miyata et al., 2004; Laxmi and Khan, 2013), and patients' families (Yun et al., 2010) approach disclosure (Lee and Wu, 2002; Fujimori and Uchitomi, 2009).

Globally, cancer is a leading cause of death, responsible for $8 \%$ mortality worldwide and about $6 \%$ of mortality in India (Ferlay et al., 2010). India has a lower cancer incidence rate compared with more developed countries but the absolute number of deaths due to cancer is large (0.5 million in 2003) (Dikshit et al., 2012). As India's population lives longer and grows older, the burden of cancer is expected to grow further to nearly 1.2 million deaths by 2035 (Ferlay et al., 2015). Physicians will be increasingly called upon to disclose a diagnosis of cancer to their patients.

Chaturvedi and colleagues $(2009 ; 2014)$ have reviewed socio-cultural factors affecting disclosure in India, and there are empirical studies that examine disclosure from the perspective of Indian doctors (Kumar et al., 2009) and patients (Laxmi and Khan, 2013). However, there is little research regarding patients' and families' perspectives on the role of family members in disclosure in India. To address this gap in the literature, we examined the disclosure preferences of cancer patients and their families. Specifically, we investigated patient preferences regarding cancer disclosure, and their perception of the role of their family members in this disclosure, and asked how patient preferences differed from those of their family members.

\section{Materials and Methods}

The current study was conducted with patients diagnosed with cancer being treated at the Medical Oncology department of a 1200 bed tertiary teaching and referral hospital in Bangalore, South India. All participants were over age 18 , accompanied by a family member, and included both inpatients and outpatients. About half were recruited after scheduled chemotherapy sessions, and the other half following general consultations. One hundred and fifty $(\mathrm{n}=150)$ patient-family member pairs were enrolled in the study. The interviews were conducted between August and November, 2011.

The consulting medical oncologist introduced each patient and accompanying family member to the researcher, who would explain the purpose of the study and obtain informed consent. Guided by a standardized questionnaire, structured interviews were conducted by the researcher in a language of the participant's preference (English, Kannada, Hindi, and Tamil). Interviews typically took about 30 minutes, and were conducted in a private setting. Patients and family members were interviewed separately about their preferences regarding the specifics of the cancer disclosure, doctor's behavior, and the role of family members. Although similar in content, differently worded questionnaires were used for patients who knew their diagnosis, did not know their diagnosis, and the accompanying family member. The questionnaire was constructed with appropriate prompts for the interviewer to skip questions with the potential to inadvertently disclose the diagnosis to patients that did not know their diagnosis.

During the interview, answers were marked into the questionnaire by the interviewer. All respondents answered questions within the provided options, and none used the open-ended 'other' option that was included when appropriate.

\section{Statistical methods}

The questionnaires were coded and all data were entered into a database for analysis. We report descriptive statistics of patients that participated in the study and identified differences between those who knew and did not know the diagnosis. Subsequently, we conducted a comparative analysis to identify differences among the patient group that had wanted or not wanted disclosure of their cancer diagnosis. These analyses were conducted with standard descriptive methods. Differences between means and proportions were assessed by chi-square test. All data were analyzed using SPSS v.16.0 (Chicago, IL).

\section{Results}

\section{Patient characteristics}

Participants ranged in age from 18 to 88 , with a mean age of 53.5 years. More than half the participants were male $(60 \%)$, had some college education $(68 \%)$, were employed $(75 \%)$, and were from a urban area $(89 \%)$. Less than half (39\%) were interviewed in English. The majority of patients $(n=127,85 \%)$ were aware of their diagnoses. Compared to participants who did not know their diagnoses $(\mathrm{n}=23,15 \%)$, participants who knew their diagnoses were more likely to have some college education $\left(\chi^{2}=17.62, p<0.001\right)$ and be English-proficient $\left(\chi^{2}=10.23, p<0.01\right)$, but did not significantly differ in

Table 1. Demographic Characteristics of Cancer Patients who had Wanted and not Wanted Disclosure

\begin{tabular}{lcccc}
\hline Characteristic & $\begin{array}{c}\text { All } \\
\mathrm{N}=127\end{array}$ & $\begin{array}{c}\text { PWD } \\
\mathrm{n}=91\end{array}$ & $\begin{array}{c}\text { PNWD } \\
\mathrm{n}=36\end{array}$ & $\chi^{2}$ \\
\hline $\begin{array}{l}\text { Gender } \\
\quad \text { Female }\end{array}$ & $52(41)$ & $39(43)$ & $13(36)$ & $\mathrm{ns}$ \\
$\quad$ Male & $75(59)$ & $52(57)$ & $23(64)$ & \\
$\begin{array}{l}\text { Education } \\
\quad 0-10 \text { years }\end{array}$ & $32(25)$ & $17(19)$ & $15(42)$ & \\
$\quad$ 11+ years & $95(75)$ & $74(81)$ & $21(58)$ & \\
Employment status & & & & $\mathrm{ns}$ \\
$\quad$ Unemployed & $33(26)$ & $23(25)$ & $10(28)$ & \\
$\quad$ Employed & $94(74)$ & $68(75)$ & $26(72)$ & \\
Interview language & & & & $12.4^{*}$ \\
$\quad$ English & $56(44)$ & $49(54)$ & $7(19)$ & \\
$\quad$ Native Indian ${ }^{1}$ & $71(56)$ & $42(46)$ & $29(81)$ & \\
$\quad$ Mean (SD) & & & & \\
Age & $52(17)$ & $52(18)$ & $52(11)$ & ns \\
\hline *p<.05; 'Kannada, Hindi, or Tamil; PWD: Patients who had wanted \\
disclosure; PNWD: Patients who had not wanted disclosure
\end{tabular}
disclosure; PNWD: Patients who had not wanted disclosure 
Breaking Bad News: Patient Preferences and the Role of Family Members when Delivering a Cancer Diagnosis

terms of age, gender, or employment status. Since minimal further information was obtained from participants who did not know their diagnosis, the following results are based on those who knew their diagnosis $(n=127)$.

Participants who knew their diagnosis ranged in age from 18 to 88 , with a mean age of $51.8(\mathrm{SD}=16.6)$. More than half were male $(59 \%)$, had some college education $(75 \%)$, were employed $(74 \%)$, and were from an urban area $(91 \%)$. Less than half $(44 \%)$ were interviewed in English. A majority of participants $(\mathrm{n}=91,72 \%)$ had wanted disclosure (PWD), while the remainder $(\mathrm{n}=36$, $28 \%$ ) had not wanted disclosure (PNWD). PWD did not differ from PNWD in terms of age, gender, or employment status, but were significantly more likely to have some college education $\left(\chi^{2}=7.23, \mathrm{p}<0.01\right)$, and be Englishproficient $\left(\chi^{2}=12.38, \mathrm{p}<0.001\right)($ Table 1$)$.

\section{Preferences regarding disclosure}

The main reasons that PWD had wanted disclosure were to not underestimate the gravity of the disease (57\%) and to make good treatment choices (51\%). A slight majority had wanted to know the diagnosis as soon as possible $(57 \%)$. On the other hand, the main reason that PNWD had not wanted disclosure was because they felt the knowledge would make them lose hope (56\%). Many (69\%) wished they had been told that they had an 'illness' or a 'growth', rather than cancer. A slight majority had wanted to know their diagnosis at a later time, when they were better prepared for it $(56 \%)$.

There were no significant differences between the

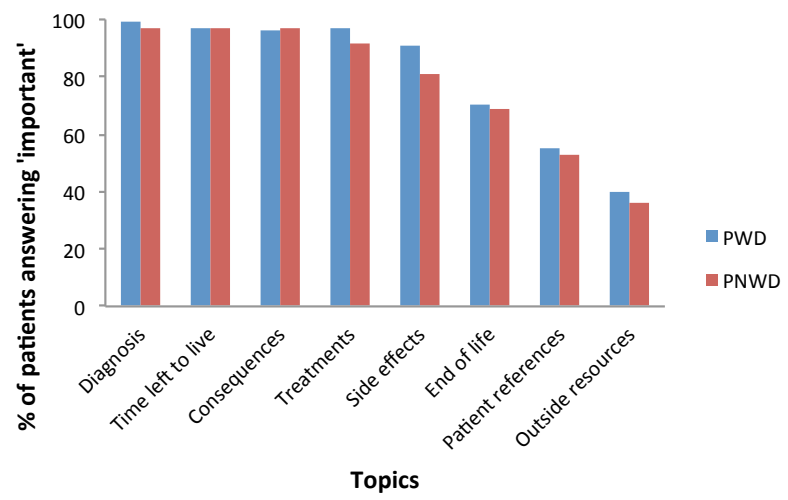

Figure 1. Patient perceptions of the importance of information

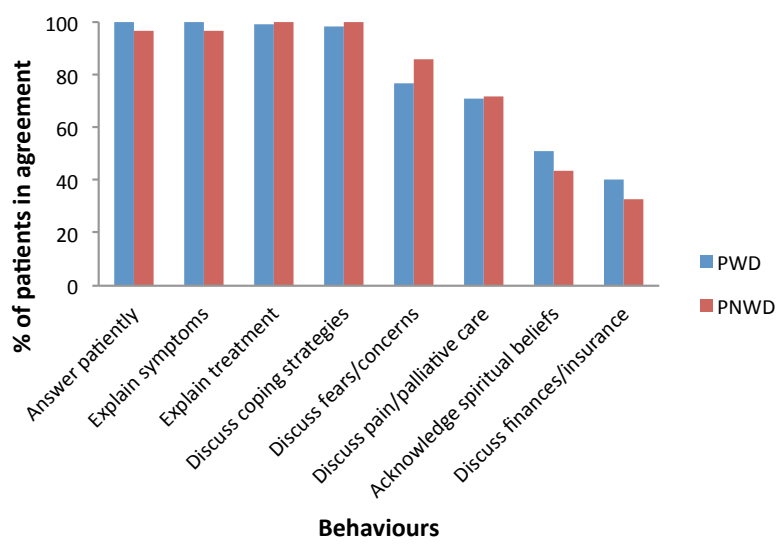

Figure 2. Patient Preferences of Doctor's Behavior two patient groups with regards to their preferences for information about their condition, with the vast majority of all patients noting that it was important to find out about medical issues, including diagnosis (98\%), prognosis (97\%), consequences and complications (96\%), treatment options $(95 \%)$, side effects $(88 \%)$, and end of life care $(70 \%)$, rather than non-medical issues, such as patient references $(54 \%)$ or other resources $(39 \%)$ (Figure 1). This was consistent with their preferences regarding the doctor's behavior, which patients agreed should be focused on explaining medical aspects of the disease such as treatment (99\%), symptoms (99\%), or coping strategies (98\%) rather than discussing non-medical aspects such as spiritual beliefs $(50 \%)$ or financial/insurance status (38\%) (Figure 2).

\section{Patient preferences regarding role of family members}

Most patients $(\mathrm{n}=114,90 \%)$ wanted their family members to be involved in some capacity in disclosure. The majority of patients $(n=80,63 \%)$ preferred that the physician disclose to their family first, a preference held significantly more strongly by PNWD (89\%) than PWD $(53 \%)\left(\chi^{2}=14.45, \mathrm{p}<0.05\right)$.

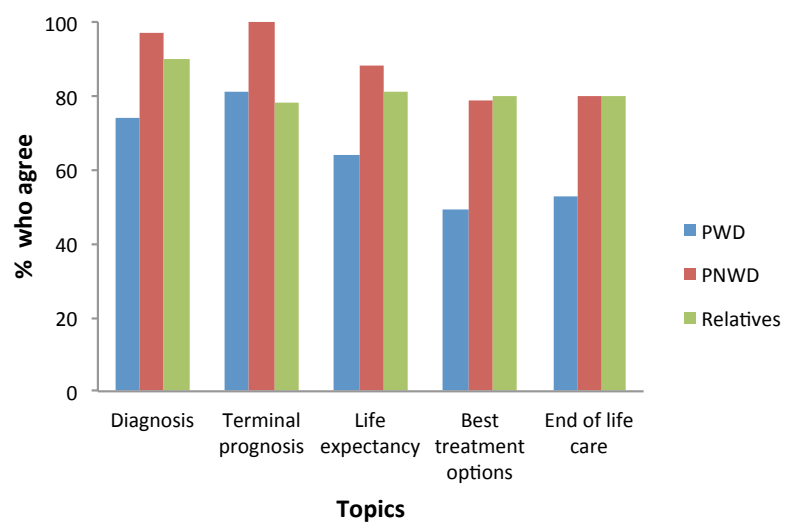

Figure 3. For those respondents who want initial disclosure to family: Preferences involving allowing family members to make decisions regarding disclosure and treatment

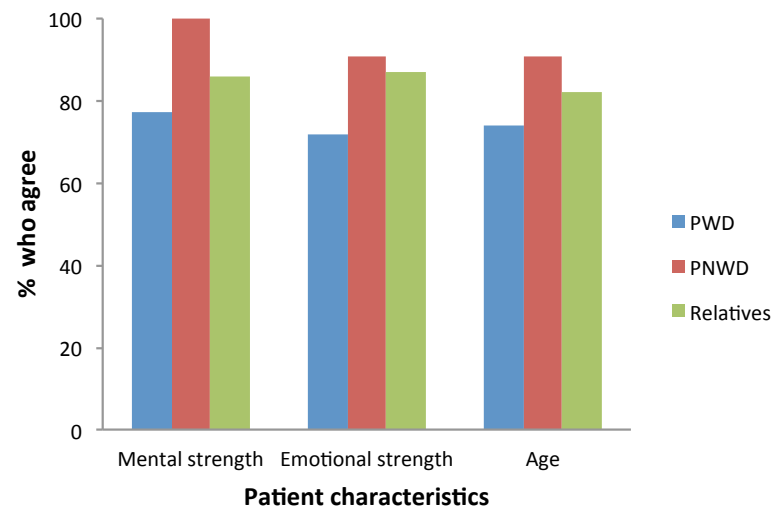

Figure 4. For those respondents who want initial disclosure to family: Preferences involving allowing family members to judge patient characteristics as a basis for disclosure 
Of those patients who preferred initial disclosure to the family $(n=80)$, the majority of patients agreed that family members should be able to request a doctor to withhold information regarding their condition and make decisions regarding treatment on behalf of the patient (Figure 3). These preferences were held significantly more strongly by PNWD than PWD (with values ranging from $\chi^{2}=4.21$ regarding end of life care to $\chi^{2}=7.28$ regarding treatment options, all $\mathrm{p}<0.05)$. The majority of this group of patients also agreed that family members were in a better position to judge the patient's personal characteristics (age) and circumstances (mental and emotional strength) as a basis for disclosure to the patient than the patient themselves (Figure 4). These preferences, too, were held more strongly by PNWD than PWD (with values ranging from $\chi^{2}=3.44$ for age, to $\chi^{2}=8.96$, for mental strength, all $\mathrm{p}<0.05)$.

\section{Comparison of patient and family member preferences}

Family members were split between wanting the patient to know and not know the diagnosis (50\%). However, the preferences of the family members generally aligned with those of the patient: when patients had wanted disclosure, a majority of family members $(59 \%)$ wished for the patient to be disclosed to as well, and when patients had not wanted disclosure, a majority of family members (72\%) did not want disclosure to the patient either. Family members were significantly more likely to want disclosure for patients who were employed $\left(\chi^{2}=\right.$ $7.97, \mathrm{p}<0.05)$; however, the education status and language proficiency of the patient were not related to the family members' disclosure preferences.

The majority of family members agreed that they should be able to request a doctor to withhold information regarding the condition and make decisions regarding treatment on behalf of the patient (see Figure 3), as they too agreed that they were in a better position than the patient to judge the patient's personal characteristics and circumstances (see Figure 4).

\section{Discussion}

In this sample of patients that sought care at a tertiary medical center, most patients strongly preferred for their family to be involved in the disclosure of their cancer diagnosis, and were interested in more information about their medical condition. However, despite this similarity, patients that had wanted and not wanted disclosure differed in significant ways. Compared to the latter group, the former wanted disclosure about their disease early and directly from the doctor, and were more likely to demand some measure of autonomy and control with respect to family involvement in disclosure. Family members were equally split in their preferences regarding disclosure to the patient, but they generally aligned themselves with the preference of the patient. The findings of the current study confirm and expand on previous studies in the area of patient and family member preferences on disclosure (Yun et al., 2004; Miyata et al., 2005; Laxmi and Khan, 2013; Chaturvedi et al., 2014), and add to our understanding of the preferences of patients and family members regarding the disclosure of a cancer diagnosis in India.

Overall, a significant proportion of interviewed patients did not know their diagnosis, although it is unclear why this is the case. While patients may independently decide to remain uninformed, it is possible that restrictions by family members (Chaturvedi et al., 2009), or limitations in the physician's communication abilities (Gattellari et al., 1999) or willingness (Tesser et al., 1971) may also result in the patient's ignorance.

Consistent with previous research conducted in a number of countries, most patients wanted to know their diagnosis (Miyata et al., 2004; Yun et al., 2004; Eng et al., 2012; Laxmi and Khan, 2013). The desire for disclosure was associated with higher education and English language proficiency. Some previous studies have found higher education to be associated with a greater desire for disclosure (Fujimori and Uchitomi, 2009; Yun et al., 2010), while others have found no relationship between the two (Yun et al., 2004; Miyata et al., 2005). In the current study, it is likely that education (and the associated English-proficiency) may have allowed patients to feel better equipped to understand their condition and make suitable medical decisions. As was the case with education, previous research on age (Miyata et al., 2005; Fujimori and Uchitomi, 2009) and gender have yielded mixed results (Yun et al., 2004; Miyata et al., 2005; Laxmi and Khan, 2013), but neither was found to be a significant factor in our study. Employment status has been found to be associated with a patient's desire for disclosure (Yun et al., 2010), but this was not found to be a significant factor in the current study.

The most compelling reasons that patients wanted disclosure was to understand the gravity of the disease and to make good treatment choices (not delay treatment, complete treatment with full adherence), and they wanted this information without delay, which is consistent with studies that have examined these factors (Benson and Britten, 1996; Miyata et al., 2004; Yun et al., 2004; Russell and Ward, 2011). The main reason that some patients did not want disclosure was that they felt that they would lose hope of recovery, which too is consistent with previous research (Akabayashi et al., 1999), and they preferred to be told, if at all, later in their treatment. In their view, the term cancer implied suffering or death, so they wished that they had been told, instead, that they had an 'illness' or a 'growth', terms which they felt was less traumatic. It is also possible that a patient who is already feeling hopeless, pessimistic, or otherwise unprepared to deal with their condition may be less willing to hear a diagnosis, rather than the reverse (Yun et al., 2004).

The majority of the patients wanted more information about their condition, consistent with past research, which suggests that most patients desire full diagnostic and prognostic information about their cancer (Eng et al., 2012; Laxmi and Khan, 2013). Unexpectedly, few differences arose between patients who had wanted and not wanted disclosure with regard to this preference. It is possible that, once aware of the diagnosis, these patients too believe that it is best to be knowledgeable about their condition and treatment. Patient preferences for information were limited to the specifics of their medical 
condition, such as diagnosis, prognosis, and treatment options; few patients were interested in non-medical issues such as accessing patient references, support groups, or other outside resources. While studies have found that such non-medical information can give patients a sense of control and reduce uncertainty (Russell and Ward, 2011), our findings are consistent with other researchers who have noted the same preference for patients to exclusively focus on current treatments and pragmatic medical issues ('getting better'), rather than the complex social and emotional ones ('feeling better') (Chaturvedi et al., 2009; Eng et al., 2012). The modern medical system may also play a role in promoting this preference: by always having another doctor's appointment, treatment, or test result to anticipate, the patient's ability or willingness to think about issues that are not directly or immediately related to the illness is diminished (The et al., 2000). It is possible that these factors may come into play with a terminal diagnosis.

Almost all patients wished for their family members to be included in the disclosure, a common preference worldwide (Benson and Britten, 1996), and one that appears to be particularly strong in certain cultural settings, including India (Akabayashi et al., 1999; Chaturvedi et al., 2009). In fact, a majority of patients preferred that the initial disclosure was made to family members rather than themselves; subsequently, patients in this group even preferred that decisions related to the disclosure were made by the family members rather than themselves. These preferences were held more strongly by patients who had not wanted disclosure than those who had.

Consistent with studies in other Asian countries, only half the family members were agreeable to disclosure to the patient, and strongly felt that they were in a better position than the patient to decide on this matter (Yun et al., 2004). This preference may be a matter of kindness and concern, since family members feel that the responsibility and burden of the patient's illness is to be borne by them (Chaturvedi et al., 2009). It may also be a matter of practicality, since the willingness of family members to agree to disclosure to the patient was significantly associated with the patient's employment status (similar to the finding in Yun et al., 2010), perhaps due to the perceived ability to pay for treatment. With respect to decision-making, the disclosure preferences of family members fell between the preferences of the two patient groups. The only exception was in the case of disclosure of a terminal prognosis: perhaps family members felt it was acceptable and even compassionate to conceal many aspects of a cancer diagnosis, but felt uncomfortable concealing such a serious and final outcome from the patient.

A potential limitation of this study is that the data are cross-sectional; a longitudinal study would allow examination of changes in patient and family member preferences over time or as a result of disease advancement. Details regarding the cancer, including disease stage or prognosis, or regarding family members, including gender, relationship to the patient, or the degree to which they are involved in patient care, were not available. It is possible that these factors could influence views relating to the cancer diagnosis. The role of family members is worth examining because their participation can both aid and impede medical care (Chaturvedi et al., 2014; Rizalar et al., 2014). Despite these limitations, the study provides useful insights into the disclosure preferences of cancer patients and their family members, and could potentially sensitize physicians involved in the care of cancer patients.

In conclusion, The current study demonstrates that patients desire disclosure and further information about their diagnosis of cancer (Laxmi and Khan, 2013), and wish to have their family involved in this process (Chaturvedi et al., 2009). The study also identifies how patients find ways of asserting their autonomy by indicating varied preferences with respect to the specifics of disclosure (degree, timing, terminology, individuals involved), as well as defining the specific role and involvement of family members. Since Indian doctors report being inadequately prepared to communicate cancer related information to patients, these findings may sensitize doctors about patient preferences, and serve to guide doctors' subsequent interactions with patients and their family members. Additional studies are required to help guide physician-patient and physician-family communication with respect to cancer care in these contexts.

\section{Acknowledgements}

This work was supported, in part, by a grant from the wellcome trust DBT India Alliance.

\section{References}

Akabayashi A, Fetters MD, Elwyn TS (1999). Family consent, communication, and advance directives for cancer disclosure: A Japanese case and discussion. J Med Ethics, 25, 296-301.

Baile WF, Buckman R, Lenzia R, et al (2000). SPIKES: A sixstep protocol for delivering bad news: Application to the patient with cancer. Oncologist, 5, 302-11.

Benson J, Britten N (1996). Respecting the autonomy of cancer patients when talking with their families: Qualitative analysis of semi-structured interviews with patients. $B M J$, 313, 729-31.

Chaturvedi SK, Loiselle CG, Chandra PS (2009). Communication with relatives and collusion in palliative care: A crosscultural perspective. Ind J Pall Care, 15, 2-9.

Chaturvedi SK, Strohschein FJ, Saraf G, Loiselle CG (2014). Communication in cancer care: Psycho-social, interactional, and cultural issues. A general overview and the example of India. Front Psychol, 5, 1332.

Dikshit R, Gupta PC, Ramasundarahettige C, et al (2012). Cancer mortality in India: a nationally representative survey. Lancet, 379, 1807-16.

Eng TC, Yaakup H, Shah SA, Jaffar A, Omar K (2009). Preferences of Malaysian cancer patients in communication of bad news. Asian Pac J Cancer Prev, 13, 2749-52.

Ferlay J, Shin HR, Bray F, et al (2010). Estimates of worldwide burden of cancer in 2008: GLOBOCAN 2008. Int J Cancer, 127, 2893-917.

Ferlay J, Soerjomataram I, Dikshit R, et al (2015). Cancer incidence and mortality worldwide: Sources, methods, and major patterns in GLOBOCAN 2012. Int J Cancer, 136, 
359-86.

Fujimori M, Uchitomi Y (2009). Preferences of cancer patients regarding communication of bad news: A systematic literature review. Jpn J Clin Onc, 39, 201-16.

Garg A, Buckman R, Kason Y (2000). Teaching medical students how to break bad news. Can Med Assoc J, 156, 1159-64.

Gattellari M, Butow PN, Tattersall MHN, Dunn SM, MacLeod CA (1999). Misunderstanding in cancer patients: Why shoot the messenger? Ann Oncol, 10, 39-46.

Ghooi RB, Deshpande SR (2012). Patients' rights in India: An ethical perspective. Ind J Med Ethics, 9, 277-81.

Grassi L, Giraldi T, Messina EG, et al (2000). Physicians' attitudes to and problems with truth-telling to cancer patients. Supp Care Cancer, 8, 40-5.

Kazdaglis GA, Arnaoutoglou C, Karypidis D, et al (2010). Disclosing the truth to terminal cancer patients: Adiscussion of ethical and cultural issues. East Mediterranean Hlth J, 16, 442-7.

Kumar M, Goyal S, Singh K, et al (2009). Breaking bad news issues: A survey among radiation oncologists. Ind $J$ Pall Care, 15, 61-6.

Laxmi S, Khan AJ (2013). Does the cancer patient want to know? Results from a study in an Indian tertiary care center. $S$ Asian J Cancer, 2, 57-61.

Lee A, Wu HY (2002). Diagnosis disclosure in cancer patients - when the family says No! Singapore Med J, 43, 533-8.

Masaki S, Ishimoto H, Asai A (2014). Contemporary issues concerning informed consent in Japan based on a review of court decisions and characteristics of Japanese culture. BMC Med Ethics, 15, 8 .

Mitchell JL (1998). Cross-cultural issues in the disclosure of cancer. Cancer Prac, 6, 153-60.

Miyata H, Tachimori H, Takahashi H, Saito T, Kai I (2004). Disclosure of cancer diagnosis and prognosis: A survey of the general public's attitudes towards doctors and family holding discretionary powers, BMC Med Ethics, 5, 7.

Miyata H, Takahashi M, Saito T, Tachimori H, Kai I (2005). Disclosure preferences regarding cancer diagnosis and prognosis: to tell or not to tell? J Med Ethics, 31, 447-51.

Rizalar S, Ozbas A, Akyolcu N, Gungor B (2014). Effect of perceived social support on psychosocial adjustment of Turkish patients with breast cancer. Asian Pac J Cancer Prev, 15, 3429-34.

Russell BJ, Ward AM (2011). Deciding what information is necessary: Do patients with advanced cancers want to know all the details? Cancer Mgmt Res, 3, 191-99.

Searight HR, Gafford J (2005). Cultural diversity at the end of life: Issues and guidelines for family physicians. Am Fam Phys, 71, 515-22.

Tesser A, Rosen S, Tesser M (1971). On the reluctance to communicate undesirable messages (the MUM effect): A field study. Psych Rep, 29, 651-4.

The AM, Hak T, Koëter G, van der Wal G (2000). Collusion in doctor-patient communication about imminent death: An ethnographic study. BMJ, 321, 1376-81.

Yun YH, Lee CG, Kim SY, et al (2004). The attitudes of cancer patients and their families toward the disclosure of terminal illness. J Clin Onc, 22, 307-14.

Yun YH, Kwon YC, Lee MK, et al (2010). Experiences and attitudes of patients with terminal cancer their family caregivers toward the disclosure of terminal illness. J Clin Onc, 28, 1950-7. 